This item was submitted to Loughborough's Research Repository by the author.

Items in Figshare are protected by copyright, with all rights reserved, unless otherwise indicated.

\title{
The influence of adiposity and acute exercise on circulating hepatokines in normal weight and overweight/obese men
}

\section{PLEASE CITE THE PUBLISHED VERSION}

https://doi.org/10.1139/apnm-2017-0639

\section{PUBLISHER}

NRC Research Press (C) The Authors

\section{VERSION}

AM (Accepted Manuscript)

\section{PUBLISHER STATEMENT}

This work is made available according to the conditions of the Creative Commons Attribution-NonCommercialNoDerivatives 4.0 International (CC BY-NC-ND 4.0) licence. Full details of this licence are available at: https://creativecommons.org/licenses/by-nc-nd/4.0/

\section{LICENCE}

CC BY-NC-ND 4.0

\section{REPOSITORY RECORD}

Sargeant, Jack, Guruprasad P. Aithal, Toshinari Takamura, Hirofumi Misu, Hiroaki Takayama, Jessica A. Douglas, Mark Turner, et al.. 2017. "The Influence of Adiposity and Acute Exercise on Circulating Hepatokines in Normal Weight and Overweight/obese Men”. figshare. https://hdl.handle.net/2134/27658. 
1 The influence of adiposity and acute exercise on circulating hepatokines in normal

2 weight and overweight/obese men

3 Jack A Sargeant ${ }^{1}$, Guruprasad P Aithal $^{2}$, Toshinari Takamura ${ }^{3}$, Hirofumi Misu ${ }^{3}$, Hiroaki

4 Takayama $^{3}$, Jessica Douglas ${ }^{1}$, Mark C Turner ${ }^{1}$, David J Stensel ${ }^{1}$, Myra A Nimmo ${ }^{4}$, David R

$5 \mathrm{Webb}^{5}$, Thomas Yates ${ }^{5}$, James A King ${ }^{1}$.

6

$7 \quad{ }^{1}$ School of Sport, Exercise and Health Sciences, Loughborough University, UK

$8{ }^{2}$ Nottingham Digestive Diseases Centre, National Institute for Health Research (NIHR)

9 Nottingham Digestive Diseases Biomedical Research Unit, Nottingham University Hospitals

10 NHS Trust and University of Nottingham, UK

$11{ }^{3}$ Department of Disease Control \& Homeostasis, Kanazawa University Graduate School of

12 Medical Sciences, Japan

$13{ }^{4}$ College of Life \& Environmental Sciences, University of Birmingham, UK

$14{ }^{5}$ Diabetes Research Centre, University of Leicester, UK

15

16 Author's email addresses

17 Jack A Sargeant - J.A.Sargeant@ lboro.ac.uk

18 Guruprasad P Aithal - Guru.Aithal@nottingham.ac.uk

19 Toshinari Takamura - ttmedfm@gmail.com

20 Hirofumi Misu - hmisu@m-kanazawa.jp

$21 \quad$ Hiroaki Takayama - htakayama@m-kanazawa.jp

22 Jessica Douglas - jessica.anne.douglas@gmail.com

23 Mark C Turner - M.C.Turner@lboro.ac.uk

24 David J Stensel - D.J.Stensel@lboro.ac.uk

25 Myra A Nimmo - M.A.Nimmo@lboro.ac.uk 
26 David R Webb - david.webb@uhl-tr.nhs.uk

27 Thomas Yates - ty20@ leicester.ac.uk

28 James A King - J.A.King@lboro.ac.uk

29

30 Address for correspondence

31 Dr James King

32 School of Sport, Exercise and Health Sciences

33 Loughborough University

$34 \quad$ Leicestershire

35 United Kingdom

36 LE11 3TU

37 Phone: $+44(0) 1509228457$

$38 \quad$ Email: J.A.King@lboro.ac.uk

39 


\section{Abstract}

41 Hepatokines are liver-secreted proteins with potential to influence glucose regulation and other metabolic parameters. This study investigated differences in adiposity status on five novel hepatokines and characterised their response to acute moderate-intensity exercise in

44 groups of normal weight and overweight/obese men.

45 Twenty-two men were recruited into normal weight and overweight/obese groups (BMI: 18.5 46 to 24.9 and 25.0 to $34.9 \mathrm{~kg} \cdot \mathrm{m}^{-2}$ ). Each completed two experimental trials, exercise and control.

47 During exercise trials, participants performed $60 \mathrm{~min}$ of moderate-intensity treadmill exercise $48\left(\sim 60 \% \quad \dot{V} \mathrm{O}_{2}\right.$ peak) and then rested for $6 \mathrm{~h}$. Participants rested throughout control trials. 49 Circulating fibroblast growth factor-21 (FGF21), follistatin, leukocyte cell-derived 50 chemotaxin 2 (LECT2), fetuin-A and selenoprotein-P (SeP) were measured throughout.

51 Fasted (resting) FGF21 and LECT2 were higher in overweight/obese individuals (129\% and $55 \% ; P \leq 0.01)$ and correlated with indices of adiposity and insulin resistance; whereas circulating follistatin was lower in overweight/obese individuals throughout trial days $(17 \%$,

$54 P<0.05)$. In both groups, circulating concentrations of FGF21 and follistatin were transiently 55 elevated after exercise for up to $6 \mathrm{~h}(P \leq 0.02)$. Circulating fetuin-A and SeP were no different between groups $(P \geq 0.19)$ and, along with LECT2, were unaffected by exercise $(P$ $57 \geq 0.06)$.

58 These findings show that increased adiposity is associated with a modified hepatokine profile, which may represent a novel mechanism linking excess adiposity to metabolic health. Furthermore, acute perturbations in circulating FGF21 and follistatin after exercise may 61 contribute to the health benefits of an active lifestyle.

62 Key words: physical activity, insulin resistance, obesity, liver, organokines 
64 Recent work characterising the hepatic proteome has identified 168 proteins which can be secreted and potentially exert endocrine-like effects in distal sites (Meex et al. 2015). A number of these 'hepatokines' are associated with measures of adiposity (Chen et al. 2011,

$67 \mathrm{Xu}$ et al. 2011, Yang et al. 2011, Lan et al. 2014) and have been shown to exert metabolic 68 effects within various central and peripheral tissues (Misu et al. 2010, Camporez et al. 2013, 69 Malin et al. 2013, Lan et al. 2014, Hansen et al. 2016b). Together, this evidence has 70 prompted suggestions that hepatokines represent a potential mechanism linking adiposity and 71 metabolic health and may be novel therapeutic targets to combat obesity-related insulin 72 resistance and associated metabolic disease.

73

74 To date, much of the research concerning hepatokine function and metabolism has focused on their direct influence on tissue-specific insulin sensitivity and systemic glucose metabolism. The most frequently studied, fibroblast growth factor-21 (FGF21), has been shown to 77 improve glucose metabolism in the liver, skeletal muscle and adipose tissue (Camporez et al. 78 2013); whilst follistatin may promote pancreatic beta cell survival and suppress circulating 79 glucagon (Hansen et al. 2016b). Other hepatokines may act to promote insulin resistance. For example, within skeletal muscle, leukocyte cell-derived chemotaxin 2 (LECT2) (Lan et al.

81 2014), selenoprotein-P (SeP) (Misu et al. 2010) and fetuin-A (Malin et al. 2013) have each

82 been shown to directly interfere with distinct aspects of glucose metabolism. Observational 83 evidence in humans has identified associations between these hepatokines and adiposity, 84 insulin resistance, ectopic lipid and the metabolic syndrome (Zhang et al. 2008, Li et al. 2010, 85 Chen et al. 2011, Xu et al. 2011, Yang et al. 2011, Choi et al. 2013, Hansen et al. 2013, 86 Okumura et al. 2013, Lan et al. 2014). However, human experimental research is now 87 required to scrutinise the pathophysiological relevance of these novel proteins in vivo. 
88 Current evidence demonstrates that exercise training reduces circulating levels of fetuin-A and FGF21, and responses correlate with improvements in insulin sensitivity and intrahepatic fat (Malin et al. 2013, 2014, Taniguchi et al. 2016). Given that single bouts of exercise transiently enhance insulin sensitivity (Sylow et al. 2017), a handful of studies have also investigated the acute influence of exercise on circulating hepatokines, speculating that modulation of the hepatokine profile may be implicated in the benefits induced. This hypothesis is strengthened by the knowledge that exercise acutely increases circulating nonesterified fatty acids (NEFA) and glucagon, and activates hepatic AMP-activated protein kinase (AMPK) (Camacho et al. 2006, Hansen et al. 2016a). Each of these have been implicated in the regulation of at least one of the hepatokines outlined above (Jung et al. 2013, Lan et al. 2014, Trepanowski et al. 2014, Hansen et al. 2016a). Whilst, these studies remain limited in number, the available evidence shows that moderate- to high-intensity aerobic exercise acutely increases circulating levels of FGF21 and follistatin (Slusher et al. 2015, Hansen et al. 2016a, 2016b), but responses may differ between normal weight and obese individuals, and between individuals with normal and dysregulated glucose metabolism (Slusher et al. 2015, Hansen et al. 2016a). Additional work is required to determine whether the FGF21 and follistatin responses to acute exercise differ between normal weight and overweight/obese individuals who are free of diagnosed metabolic disease, and whether similar responses occur in other relevant hepatokines.

The purpose of this study was two-fold. Firstly, we sought to investigate differences in adiposity status on FGF21, follistatin, LECT2, SeP and fetuin-A in normal weight and overweight/obese men. Secondly, we characterised the effect of an acute bout of moderateintensity exercise on circulating concentrations of these hepatokines in order to explore their potential role as mediators of exercise-induced improvements in glycaemic control and other 
113 metabolic parameters. We hypothesised that overweight/obese individuals would have 114 elevated circulating concentrations of hepatokines at rest and that an acute bout of moderate115 intensity exercise would beneficially alter circulating hepatokine profiles; by reducing 116 LECT2, SeP, and fetuin A, whilst increasing FGF21 and follistatin. 


\section{Materials and methods}

\section{Ethical approval and participant recruitment}

119 After receiving approval from the East Midlands NHS Research Ethics committee 120 (13/EM/0290), 22 non-smoking men were recruited equally into normal weight and 121 overweight/obese groups (BMI: 18.5 to 24.9 and 25.0 to $34.9 \mathrm{~kg} \cdot \mathrm{m}^{-2}$, respectively); providing 122 written, informed consent to participate. This sample size was chosen based on previous 123 studies that documented significant changes in hepatokines (FGF21 and follistatin) in 124 response to acute exercise; as well as differences between participant groups (normal weight 125 vs. obese and normal vs. impaired glucose regulation) (Slusher et al. 2015, Hansen et al. 126 2016a, 2016b). Participants in the current study were free of diagnosed chronic disease and 127 were not taking medications known to affect glucose or lipid metabolism, or blood pressure. 128 Participants were also 'inactive' or 'moderately active' according to the International 129 Physical Activity Questionnaire (www.ipaq.ki.se [accessed 03/01/2017]) and were weight stable in the three months prior to enrolment ( $<2 \mathrm{~kg}$ self-reported weight change).

\section{Participant pre-assessment}

133 During a pre-assessment visit, participants were screened to determine eligibility and suitability for exercise testing. Participants underwent full medical evaluation, including assessment of cardiovascular disease risk, fasted capillary blood glucose and completion of

136 the Physical Activity Readiness Questionnaire (www.csep.ca/en/publications [accessed 137 03/01/2017]). Participants were excluded if blood pressure was greater then 160/100 $\mathrm{mmHg}$, whilst resting and exercise electrocardiograms were performed to confirm the absence of established cardiac arrhythmias. Body fat was assessed using bioelectrical impedance

140 analysis (BC-418, TANITA Europe BV, Amsterdam, The Netherlands) under standardised

141 conditions and waist circumference was measured at the level of the umbilicus. 
143 On a separate occasion, participants completed a 16-min, progressive sub-maximal exercise

144 test on a motorised treadmill (Excite Med, Technogym, Italy), which was subsequently 145 followed by a ramped peak oxygen uptake test. Expired air was collected continuously 146 throughout both tests for quantification of oxygen consumption $\left(\dot{V} \mathrm{O}_{2}\right)$ and carbon dioxide 147 production $\left(\dot{V} \mathrm{CO}_{2}\right)$ using breath-by-breath indirect calorimetry (Metalyser 3B, Cortex, 148 Germany). Peak oxygen uptake ( $\dot{V} \mathrm{O}_{2}$ peak) was calculated and bivariate linear regression was 149 used to estimate the treadmill speed necessary to elicit $60 \%$ of $\dot{V} \mathrm{O}_{2}$ peak during exercise trials. 150

151 In the $48 \mathrm{~h}$ before main trials, participants refrained from strenuous physical activity, alcohol 152 and caffeine, and standardised their dietary intake using weighed records. On the evening 153 before main trials, participants were provided with a standardised meal $(3138 \mathrm{~kJ} ; 71 \%$ carbohydrate, $18 \%$ fat, $11 \%$ protein) to consume before $21: 00$ after which only water was permitted until the start of the trials.

\section{Experimental trials}

158 Participants completed two experimental trials, control and exercise, in a counterbalanced order and separated by approximately one week. On the morning of each trial, participants arrived at the laboratory at 08:30, at which point an intravenous cannula $(21 \mathrm{G}$; Venflon, 161 Becton Dickinson, Sweden) was inserted into an antecubital vein. Trials were then initiated 162 with a venous blood sample taken at 09:00 (0 h) and additional samples were collected at 1, $1631.5,2.75,4$ and $7 \mathrm{~h}$. During exercise trials, participants completed a 60-min bout of moderate-intensity treadmill exercise $\left(60 \%\right.$ of $\dot{V} \mathrm{O}_{2}$ peak) between 0 and $1 \mathrm{~h}$, and then rested in the laboratory for the remainder of the trial $(1-7 \mathrm{~h})$. Heart rate (RS100, Polar, United

166 Kingdom) and rate of perceived exertion (RPE) (Borg 1970) were recorded every $15 \mathrm{~min}$ 
167 during exercise, and expired air was collected throughout for ongoing measurement of $\dot{V} \mathrm{O}_{2}$

168 and $\dot{V} \mathrm{CO}_{2}$. If necessary, treadmill speed was adjusted to maintain the desired exercise 169 intensity. Participants rested for the entirety of control trials and samples of expired air were 170 collected between 0 and $1 \mathrm{~h}$ to quantify resting energy expenditure; allowing the calculation 171 of net energy expenditure elicited by exercise. Participants were provided with a standardised 172 breakfast $(2690 \mathrm{~kJ} ; 72 \%$ carbohydrate, $18 \%$ fat, $10 \%$ protein) and lunch $(3138 \mathrm{~kJ} ; 43 \%$ 173 carbohydrate, $25 \%$ fat, $32 \%$ protein) at 1.5 and $4 \mathrm{~h}$, respectively.

174

\section{Biochemical analyses}

176 Venous blood samples were collected into ice-cooled monovettes pre-treated with 177 anticoagulant (Sarstedt, Leicester, UK). Monovettes were spun immediately in a refrigerated 178 centrifuge $\left(4{ }^{\circ} \mathrm{C}\right)$ for $10 \mathrm{~min}$ at $2383 \times \mathrm{g}$ and the plasma supernatant was removed and 179 aliquoted for storage at $-80{ }^{\circ} \mathrm{C}$. At each time point, commercially available enzyme-linked 180 immunosorbent assays were used to measure plasma concentrations of FGF21, follistatin, 181 fetuin-A (R \& D Systems, Oxford, UK), LECT2 (MBL International, Massachusetts, USA), 182 insulin and glucagon (Mercodia, Uppsala, Sweden), whilst plasma concentrations of full183 length SeP were measured using a sol particle homogeneous immunoassay as previously 184 reported (Saito et al. 2001, Tanaka et al. 2016). The mean within-batch co-efficient of 185 variation $(\mathrm{CV})$ for these assays was $\leq 5.5 \%$. Circulating concentrations of NEFA, glucose, 186 triacylglycerol (TAG), total cholesterol, liver aminotransferases (AST, ALT) and gamma187 glutamyl transpeptidase (GGT) were analysed by enzymatic colorimetric methods using a 188 benchtop analyser (Pentra 400; HORIBA ABX Diagnostics, Montpellier, France; within 189 batch $\mathrm{CV} \leq 4.2 \%$ ). All were measured at $0 \mathrm{~h}$ only with the exception of plasma NEFA, which 190 was measured at each sample time point. Insulin resistance was assessed by HOMA-IR and 191 Adipo-IR as previously described (Matthews et al. 1985, Gastaldelli et al. 2007). 
193 Plasma volume was calculated in each whole blood sample using established equations (Dill 194 and Costill 1974). Circulating protein concentrations were corrected, as previously described 195 (Sherk et al. 2013), when plasma volume deviated significantly from baseline.

\section{Statistical analyses}

197 Unless otherwise stated, data are presented as mean \pm SD or SEM for participant 198 characteristics and outcome variables, respectively. Statistical analyses were performed using commercially available software (SPSS version 23.0, SPSS Inc., United States). Where appropriate, data were log transformed to meet assumptions for parametric statistical testing. Two-tailed, independent samples $t$-tests were used to compare differences in participant characteristics, fasted plasma protein and metabolite concentrations, and characteristics of the exercise performed between normal weight and overweight/obese groups. When parametric assumptions were not met before or after log transformation, non-parametric Wilcoxon matched-pairs signed rank test was used. Relationships between fasted hepatokine concentrations and other participant characteristics were assessed using bivariate Pearson's and Spearman's correlation analyses as appropriate. Three-way, mixed-design analysis of variance (ANOVA), consisting of two within-participant factors (trial and sample time) and one between-participant factor (group), was used to assess hepatokine responses to exercise.

210 After inspection of main effects, the three-way interaction between trial, time and group was

211 used to assess whether hepatokine responses during and after exercise, when compared to the 212 control trial, differed between normal weight and overweight/obese groups. When this was 213 not significant, the two-way interaction between trial and time was used to investigate the 214 hepatokine response to exercise in the two groups combined. Statistically significant two-way 215 interactions were investigated further with two-tailed paired samples $t$-tests to identify the 216 times at which circulating concentrations differed between control and exercise trials. Due to 
217 the sample size in this study, no correction for multiple comparisons was applied. To help 218 clarity in graphical presentation, total area under the concentration-time curve (AUC) was 219 also calculated for each experimental trial and these data were analysed statistically using 220 two-way mixed design ANOVA. Probability $(P-)$ values $\leq 0.05$ were considered statistically 221 significant. 
Results

Participant characteristics

224 Descriptive characteristics of the normal weight and overweight/obese groups can be found in 225 Table 1. By design, the overweight/obese group had higher BMI, body mass, body fat 226 percentage and waist circumference, but age was similar between groups. There was no 227 difference in absolute cardiorespiratory fitness between groups but relative fitness was 228 greater in the normal weight individuals due to their lower body mass. Fasted plasma glucose, 229 insulin and HOMA-IR were similar between groups, but fasted plasma lipids were greater in 230 the overweight/obese individuals, whilst Adipo-IR tended to be higher. There were no 231 significant differences in AST, ALT or GGT between groups (data not shown).

232

233

Insert table 1 here

234

235

Fasted plasma hepatokine concentrations and associations with metabolic variables

236 The overweight/obese individuals had greater fasted plasma concentrations of LECT2 and 237 FGF21, but fasted concentrations of follistatin, fetuin-A and SeP were similar between 238 groups (Table 1). Fasted circulating LECT2 and FGF21 were positively correlated with each 239 other $\left(r h o^{2}=36.9 \%, P=0.03\right)$, body mass, BMI, WC, body fat, NEFA, TAG, Adipo-IR and 240 glucagon $\left(r^{2} \geq 19.4 \%, P \leq 0.02\right.$ or $\left.r h o^{2} \geq 17.6 \%, P \leq 0.05\right)$, and negatively with relative $\dot{V} \mathrm{O}_{2}$ 241 peak $\left(r^{2} \geq 27.0 \%, P \leq 0.01\right)$. FGF21 was also marginally positively correlated with fasted 242 plasma glucose $\left(r^{2}=18.1 \%, P=0.048\right)$, whilst LECT2 was strongly positively correlated 243 with HOMA-IR $\left(r h o^{2}=43.2 \%, P=0.001\right)$. Significant negative correlations were found 244 between fasted concentrations of follistatin and the AST:ALT ratio $\left(r^{2}=18.5 \%, P=0.05\right)$, 245 fetuin-A and age $\left(r h o^{2}=25 \%, P=0.02\right)$, and between fasted concentrations of SeP and ALT 
$246\left(r^{2}=19.4 \%, P=0.04\right)$. Further details of all significant correlations can be found in

247 supplementary material.

248

\section{Exercise characteristics}

250 Participants in the normal weight group exercised at a greater treadmill speed due to their 251 higher relative cardiorespiratory fitness. However, the relative intensity of the exercise 252 performed was similar between groups (Table 2). Consequently, given the higher energy cost 253 of exercise in the overweight/obese group as a result of their higher body mass, the net 254 energy expenditure during exercise trials was similar between groups $(P=0.98)$. During the 255 exercise trials, there was a significant reduction in plasma volume immediately post-exercise 256 irrespective of group ( 0 vs 1 h: $58.5 \pm 0.7$ vs. $53.7 \pm 0.7 \% ; P<0.01$ ), which returned to 257 baseline by $1.5 \mathrm{~h}$.

\section{Circulating hepatokine responses to exercise}

262 Plasma FGF21 concentrations were higher in the overweight/obese group, irrespective of trial or time (Figure 1a; $P=0.003)$, but there was no interaction between trial, time and group ( $P$ $264=0.19$ ). With groups combined, there was a significant two-way interaction between trial and 265 time for circulating FGF21 (Figure 2a; $P<0.001$ ), and post-hoc analyses revealed that 266 circulating concentrations were significantly higher at $1,1.5$ and $4 \mathrm{~h}$ in the exercise trial, 267 compared with control (all $P \leq 0.005$ ). Accordingly, the total AUC for FGF21 was significantly greater in the overweight/obese compared with the normal weight individuals

269 (Figure $1 \mathrm{~b} ; P=0.003$ ), and in the exercise versus the control trials (Figure $2 \mathrm{~b} ; P=0.003$ ). 270 However, there was no interaction between group and trial $(P=0.65)$. 
272 Circulating follistatin was lower in the overweight/obese versus the normal weight group

273 (Figure 1c; $P=0.05)$, but the interaction between trial, time and group was not significant $(P$ $274=0.94)$. In the whole study population, there was a significant two-way interaction between 275 trial and time for plasma follistatin (Figure 2c; $P=0.001$ ). Post-hoc analyses identified 276 significantly higher concentrations at $2.75,4$ and $7 \mathrm{~h}$ in the exercise trial $(P \leq 0.02)$. Similarly, 277 the total AUC for follistatin was significantly lower in the overweight/obese group (Figure 1d; $278 P=0.05$ ) and greater in the exercise trials (Figure $2 \mathrm{~d} ; P<0.01$ ), but there was no interaction 279 between group and trial $(P=0.41)$.

280

281 Circulating LECT2 was higher in the overweight/obese group versus the normal weight 282 group, (Figure 1e; $P=0.009)$, but there was no interaction between trial, time and group $(P=$ 283 0.38). For plasma concentrations of LECT2, the two-way interaction between trial and time 284 in the whole study population was also not significant (Figure $2 \mathrm{e} ; P=0.06$ ). The total AUC 285 analyses for LECT2 mirrored these results with a significantly greater AUC in the 286 overweight/obese group (Figure 1f; $P<0.01$ ), but no significant difference between the 287 control and exercise trials (Figure 2f; $P=0.07)$ and no interaction between group and trial $(P$ $288=0.45)$.

289

290 Circulating concentrations of fetuin-A and SeP were similar between groups (Figures $1 \mathrm{~g}$ and $2911 \mathrm{i} ; P \geq 0.20)$ and there were no interactions between trial, time and group $(P \geq 0.07)$. 292 Furthermore, with groups combined, there were no interactions between trial and time 293 (Figures $2 \mathrm{~g}$ and $2 \mathrm{i} ; P \geq 0.11$ ). In accordance, the total AUC for fetuin-A (Figures $1 \mathrm{~h}$ and $2 \mathrm{~h}$ ) 294 and SeP (Figures $1 \mathrm{j}$ and $2 \mathrm{j}$ ) were similar between groups and between trials, and there were 295 no significant interactions (all $P \geq 0.17$ ). 


\section{Insert figure 2}

Circulating responses of NEFA, glucagon and insulin to exercise

302

Despite differences in the fasted state, circulating concentrations of NEFA were similar between groups throughout experimental trials $(P=0.09)$, and there was no significant interaction between trial, time and group $(P=0.13)$. However, with groups combined there was a significant two-way interaction between trial and time (Figure $3 \mathrm{a} ; P<0.001$ ) and posthoc analyses revealed significantly higher concentrations of NEFA in the exercise trial at 1 , $1.5,2.75$ and $7 \mathrm{~h}(P \leq 0.04)$.

308

Circulating concentrations of glucagon, insulin and glucagon to insulin ratio were also similar

310 between groups $(P \geq 0.27)$, and the three-way interactions between trial, time and group were

311 not significant for any of these outcomes $(P \geq 0.16)$. However, in the whole study population, 312 there were significant two-way interactions between trial and time for all of glucagon, insulin 313 and the glucagon to insulin ratio (Figure $3 \mathrm{~b}-\mathrm{d} ; P \leq 0.03$ ). Post-hoc tests revealed the glucagon 314 to insulin ratio was significantly greater at 1 and $1.5 \mathrm{~h}$ in the exercise trial when compared to 315 the control trial $(P \leq 0.02)$. This was primarily driven by significantly higher concentrations 316 of glucagon $(P \leq 0.01)$ and occurred despite significantly higher concentrations of insulin at $3171.5 \mathrm{~h}$ in the exercise trial $(\mathrm{P}=0.02)$. Glucagon remained elevated in the exercise trial at 2.75 $\mathrm{h}(\mathrm{P}=0.03)$ but the consequential increase in the glucagon to insulin ratio was not 319 statistically significant $(\mathrm{P}=0.08)$. 
Insert figure 3 


\section{Discussion}

323 This study investigated the impact of adiposity and acute exercise on five candidate

324 hepatokines which have been identified as novel circulating proteins linking the liver and 325 peripheral metabolism. Our findings suggest that circulating levels of LECT2, FGF21 and 326 follistatin are modulated by adiposity and are associated with various anthropometric 327 measurements and biomarkers of metabolic health. Additionally, our findings show that 328 circulating levels of FGF21 and follistatin are transiently elevated after a single bout of 329 moderate-intensity exercise, and these responses are preserved in overweight/obese 330 individuals. These responses may help mediate the favourable metabolic impact of exercise, 331 but further research is needed to assess causality.

332

333 Previous reports have shown increased LECT2 in obese individuals, with or without nonalcoholic fatty liver disease (NAFLD), in two large Japanese cohorts (Okumura et al. 2013, Lan et al. 2014). The current study is the first, however, to show that LECT2 is 336 elevated in European men that are overweight/obese and correlates with BMI in a population 337 of normal weight, overweight and obese individuals. In mice, hepatic expression and 338 circulating concentrations of LECT2 are negatively regulated by hepatic AMPK (Lan et al. 339 2014). Furthermore, eight weeks of high-fat overfeeding increased circulating concentrations 340 of LECT2, alongside increases in body mass (Lan et al. 2014). As such, circulating LECT2 341 may be increased in overweight and obese individuals due to chronic reduction of hepatic

342 AMPK activity resulting from sustained energy surplus. In agreement with previous studies 343 (Okumura et al. 2013, Lan et al. 2014), we show significant associations between LECT2 and 344 fasted plasma insulin and HOMA-IR, whilst we also report, for the first time, significant 345 correlations between fasted concentrations of LECT2, NEFA and Adipo-IR. LECT2 has been 346 shown to inhibit insulin signalling in $\mathrm{C} 2 \mathrm{C} 12$ myotubes via activation of Jun $\mathrm{NH}_{2}$-terminal 
347 kinase (JNK) (Lan et al. 2014) but its effects on other peripheral tissues, including hepatic 348 and adipose tissues, warrant further investigation.

350 Previous reports have shown that fasted concentrations of FGF21 are elevated in obese 351 individuals with normal or dysregulated glucose metabolism (Zhang et al. 2008, Chen et al. 352 2011). Our findings are in agreement with these studies and show that FGF21 is also 353 increased in individuals that are overweight/obese. Hepatic expression and secretion of 354 FGF21 is increased during periods of starvation via the activation of peroxisome proliferator355 activated receptor alpha (PPAR- $\alpha$ ) by circulating NEFA (Badman et al. 2007). Plasma 356 concentrations of NEFA are also increased with obesity (Boden 2008), offering a potential 357 mechanism to explain the increased FGF21 concentrations seen in the present study. In support of this, we report elevated fasted concentrations of NEFA in the overweight/obese group and a strong positive correlation between circulating concentrations of FGF21 and NEFA. Alternatively, a state of 'FGF21 resistance' may also result in elevated concentrations of FGF21 group (Potthoff et al. 2012) but it was beyond the scope of this study to investigate this hypothesis.

Despite no statistically significant difference in the fasted state, we showed lower concentrations of follistatin in the overweight/obese group throughout trial days. Our findings are consistent with previous data which identified lower follistatin levels in obese individuals with T2DM (Ueland et al. 2012), yet contrast those of Hansen et al. (2013) who identified higher follistatin in T2DM patients. The reasons for these discrepancies are not clear at this time and further work is therefore needed to more fully understand the metabolism of follistatin in health and disease. 
372 We report no differences between groups in concentrations of fetuin-A or SeP either in the 373 fasted state or throughout trial days. This may suggest that the development of metabolic 374 complications, and not adiposity per se, may be required to disrupt fetuin-A and SeP

375 metabolism. Previous research has found no independent effect of obesity on fetuin-A 376 concentrations (Obuchi et al. 2014), whilst studies reporting differences in SeP have recruited 377 individuals with NAFLD or dysregulated glucose metabolism (Yang et al. 2011, Choi et al. 378 2013).

379

380 In the current study we demonstrate that circulating concentrations of FGF21 are increased 381 immediately after an acute 60-min bout of moderate-intensity aerobic exercise, peaking 30 382 min after the cessation of exercise, and remaining elevated for up to $3 \mathrm{~h}$. A similar, albeit 383 delayed, increase in circulating follistatin also occurred. These findings support previous studies showing that FGF21 and follistatin are increased with acute aerobic exercise (Slusher et al. 2015, Hansen et al. 2016a, 2016b). FGF21 and follistatin production are positively 386 regulated by the glucagon to insulin ratio (Hansen et al. 2016a, 2016b), whilst FGF21 may 387 also be increased via activation of PPAR- $\alpha$ by circulating NEFA (Kim et al. 2013, Hansen et 388 al. 2016a). The systemic glucagon to insulin ratio and circulating NEFA were both elevated in response to exercise in the present study. FGF21 improves glucose metabolism in skeletal muscle, adipose tissue and the liver, whilst follistatin may promote pancreatic beta cell 391 survival, reduce circulating glucagon and preserve skeletal muscle mass (Camporez et al. 392 2013, Hansen et al. 2016b). Transient increases in the circulating levels of these hepatokines 393 may represent potential mechanisms which contribute to the short-term improvements in 394 glycaemic control after acute exercise (Sylow et al. 2017). 
In the current study the responses of circulating FGF21 and follistatin to exercise were 397 similar in both normal weight and overweight/obese participants. It has been previously shown that the FGF21 and follistatin responses to acute exercise are blunted in individuals with T2DM, and this may be the result of differences in the exercise-induced changes in 400 circulating NEFA and the glucagon to insulin ratio (Hansen et al. 2016a). Furthermore, the 401 response of FGF21 to 30 min of exercise at $75 \% \dot{V} \mathrm{O}_{2}$ peak has been shown to be reduced in 402 obese individuals compared with healthy, normal weight controls (Slusher et al. 2015). 403 Notably, although the participants in the study by Slusher and colleagues (Slusher et al. 2015) 404 were reportedly healthy, the obese group had a mean HOMA-IR of 4.36, which approaches 405 the 5.13 threshold previously used to distinguish insulin resistant individuals (Wildman et al. 406 2008). The participants in the current study were free from chronic disease and fasted plasma 407 glucose, insulin and HOMA-IR suggested they were not insulin resistant. It could, therefore, 408 be speculated that exercise-induced increases in circulating FGF21 and follistatin are 409 maintained in overweight/obese individuals with preserved glycaemic control but not once a 410 degree of insulin resistance has developed. Notably, the exercise-induced changes in NEFA 411 and the glucagon to insulin ratio were no different between groups in the current study. This speculative hypothesis, however, should be tested further.

414 The current study is the first to investigate the acute effects of aerobic exercise on fetuin-A, 415 LECT2 and SeP, but these hepatokines were unaffected by exercise. Fetuin-A, LECT2 and 416 SeP are all negatively regulated by hepatic AMPK (Jung et al. 2013, Lan et al. 2014, 417 Trepanowski et al. 2014) which is activated by exercise in an intensity-dependent manner 418 (Camacho et al. 2006). It may be that higher intensity exercise is required to acutely modulate 419 fetuin-A, LECT2 or SeP (Trepanowski et al. 2014), or that repeated bouts of exercise are 420 required to elicit benefits; as shown previously for fetuin-A (Malin et al. 2013, 2014). 
422 This study is not without limitation. Most prominently, this trial was conducted using a 423 relatively small sample of normal weight and overweight/obese men. Given the lack of prior 424 evidence we were unable to determine a priori whether our sample size was sufficient for all 425 of our outcomes. The novel data presented in this manuscript may, however, be utilised to 426 inform power calculations for future studies, particularly those investigating the effects of 427 acute exercise on LECT2, SeP and fetuin-A. Our results also cannot be generalised to 428 individuals with chronic metabolic disease or women. Notably, we did not directly measure 429 intrahepatic fat or insulin sensitivity in the current study. These are important considerations 430 because the development of metabolic disease may influence hepatokine metabolism. 431 Furthermore, the heightened propensity for fatty liver development in men, and the potential 432 metabolic influence of sex hormones, underscores the necessity for additional research to be 433 undertaken in women.

434

435 In conclusion, this study has identified higher circulating concentrations of FGF21 and 436 LECT2, and lower follistatin, in overweight/obese men when compared to normal weight 437 individuals. Moreover, circulating FGF21 and follistatin are acutely increased after moderate438 intensity aerobic exercise, and this beneficial shift in hepatokine profile is similar in both 439 groups. These data provide new information regarding the effect of adiposity on the 440 metabolism of several novel hepatokines and supports evidence for a potential role of FGF21 441 and follistatin in the metabolic response to exercise. Additional work is needed to better 442 understand the interaction between these novel proteins, obesity and chronic disease; as well 443 as to better define their interaction with exercise and other metabolic perturbations. 
444 Acknowledgements

445 We would like to thank all study participants for dedicating their time and energy to this 446 study. We would also like to thank Dr Andrew Jackson and Dr Philip Cordery for their 447 assistance during the biochemical analyses. This research was supported by the NIHR 448 Leicester Biomedical Research Centre. The views expressed are those of the authors and not 449 necessarily those of the NHS, the NIHR or the Department of Health.

450

451 Disclosures

452 The authors declare no conflicts of interest.

453

454 


\section{References}

Badman, M.K., Pissios, P., Kennedy, A.R., Koukos, G., Flier, J.S., and Maratos-Flier, E. 2007. Hepatic fibroblast growth factor 21 is regulated by PPAR $\alpha$ and is a key mediator of hepatic lipid metabolism in ketotic states. Cell Metab. 5(6): 426-437. doi:10.1016/j.cmet.2007.05.002.

Boden, G. 2008. Obesity and free fatty acids (FFAs). Endocrinol. Metab. Clin. North. Am. 37(3): 1-12. doi:10.1016/j.ecl.2008.06.007.Obesity.

Borg, G. 1970. Percieved exertion as an indicator of somatic stress. Scand. J. Rehab. Med. 2(2): 92-98.

Camacho, R.C., Donahue, E.P., James, F.D., Berglund, E.D., Wasserman, D.H., Berglund, D., et al. 2006. Energy state of the liver during short-term and exhaustive exercise in C57BL/6J mice. Am. J. Physiol. Endocrinol. Metab. 290(3): 405-408. doi:10.1152/ajpendo.00385.2005.

Camporez, J.P.G., Jornayvaz, F.R., Petersen, M.C., Pesta, D., Guigni, B.A., Serr, J., et al. 2013. Cellular mechanisms by which FGF21 improves insulin sensitivity in male mice. Endocrinology, 154(9): 3099-3109. doi:10.1210/en.2013-1191.

Chen, C., Cheung, B.M.Y., Tso, A.W.K., Wang, Y., Law, L.S.C., Ong, K.L., et al. 2011. High plasma level of fibroblast growth factor 21 is an independent predictor of type 2 diabetes: A 5.4-year population-based prospective study in Chinese subjects. Diabetes Care, 34(9): 2113-2115. doi:10.2337/dc11-0294.

Choi, H.Y., Hwang, S.Y., Lee, C.H., Hong, H.C., Yang, S.J., Yoo, H.J., et al. 2013. Increased selenoprotein $\mathrm{P}$ levels in subjects with visceral obesity and nonalcoholic fatty liver disease. Diabetes Metab. J. 37(1): 63-71. doi:10.4093/dmj.2013.37.1.63.

Dill, D.B., and Costill, D.L. 1974. Calculation of percentage changes in volumes of blood, 
Gastaldelli, A., Cusi, K., Pettiti, M., Hardies, J., Miyazaki, Y., Berria, R., et al. 2007. Relationship between hepatic/visceral fat and hepatic insulin resistance in nondiabetic and type 2 diabetic subjects. Gastroenterology, 133(2): 496-506. doi:10.1053/j.gastro.2007.04.068.

Hansen, J., Rinnov, A., Krogh-Madsen, R., Fischer, C.P., Andreasen, A.S., Berg, R.M.G., et 485 al. 2013. Plasma follistatin is elevated in patients with type 2 diabetes: relationship to hyperglycemia, hyperinsulinemia and systemic low-grade inflammation. Diabetes. Metab. Res. Rev. 29(6): 463-472. doi:10.1002/dmrr.

Hansen, J., Pedersen, B.K., Xu, G., Lehmann, R., Weigert, C., and Plomgaard, P. 2016a. Exercise-induced secretion of FGF21 and follistatin are blocked by pancreatic clamp and impaired in type 2 diabetes. J. Clin. Endocrinol. Metab. 101(7): 2816-2825. doi:10.1210/jc.2016-1681.

Hansen, J., Rutti, S., Arous, C., Clemmesen, J.O., Secher, N.H., Drescher, A., et al. 2016b. 493 Circulating follistatin is liver-derived and regulated by the glucagon-to-insulin ratio. J. Clin. Endocrinol. Metab. 101(2): 550-560. doi:10.1210/jc.2015-3668.

Jung, T.W., Choi, H.Y., Lee, S.Y., Hong, H.C., Yang, S.J., Yoo, H.J., et al. 2013. Salsalate 496 and adiponectin improve palmitate-induced insulin resistance via inhibition of selenoprotein $\mathrm{P}$ through the AMPK-FOXO1 $\alpha$ pathway. PLoS One, 8(6): 1-9. doi:10.1371/journal.pone.0066529.

Kim, K.H., Kim, S.H., Min, Y.-K., Yang, H.-M., Lee, J.-B., and Lee, M.-S. 2013. Acute exercise induces FGF21 expression in mice and in healthy humans. PLoS One, 8(5): e63517. doi:10.1371/journal.pone.0063517.

502 Lan, F., Misu, H., Chikamoto, K., Takayama, H., Kikuchi, A., Mohri, K., et al. 2014. LECT2 
functions as a hepatokine that links obesity to skeletal muscle insulin resistance. Diabetes, 63(5): 1649-1664.

Li, H., Fang, Q., Gao, F., Fan, J., Zhou, J., Wang, X., et al. 2010. Fibroblast growth factor 21 levels are increased in nonalcoholic fatty liver disease patients and are correlated with hepatic triglyceride. J. Hepatol. 53(5): 934-940. doi:10.1016/j.jhep.2010.05.018.

508

509

510

511

512

513

514

515

516

517

518

519

520

521

522

523

524

525

526

Malin, S.K., Mulya, A., Fealy, C., Haus, J., Pagadala, M., Scelsi, A., et al. 2013. Fetuin-A is linked to improved glucose tolerance after short-term exercise training in nonalcoholic fatty liver disease. J. Appl. Physiol. 115(7): 988-994. doi:10.1152/japplphysiol.00237.2013.

Malin, S.K., Del Rincon, J.P., Huang, H., and Kirwan, J.P. 2014. Exercise-induced lowering of fetuin-a may increase hepatic insulin sensitivity. Med. Sci. Sports Exerc. 46(11): 2085-2090. doi:10.1249/MSS.0000000000000338.

Matthews, D.R., Hosker, J.R., Rudenski, A.S., Naylor, B.A., Treacher, D.F., and Turner, R.C. 1985. Homeostasis model assessment: insulin resistance and $\beta$-cell function from fasting plasma glucose and insulin concentrations in man. Diabetologia, 28(7): 412-419.

Meex, R.C., Hoy, A.J., Morris, A., Brown, R.D., Lo, J.C.Y., Burke, M., et al. 2015. Fetuin B is a secreted hepatocyte factor linking steatosis to impaired glucose metabolism. Cell Metab. 22(6): 1078-1089. doi:10.1016/j.cmet.2015.09.023.

Misu, H., Takamura, T., Takayama, H., Hayashi, H., Matsuzawa-Nagata, N., Kurita, S., et al. 2010. A liver-derived secretory protein, selenoprotein P, causes insulin resistance. Cell Metab. 12(5): 483-495. doi:10.1016/j.cmet.2010.09.015.

Obuchi, A., Adachi, H., Enomoto, M., Fukami, A., Kumagai, E., Nakamura, S., et al. 2014. High plasma fetuin-A levels are associated with metabolic syndrome among males but not females in a Japanese general population. Diabetes Res. Clin. Pract. 106(1): 128-135. 
doi:10.1016/j.diabres.2014.07.002.

528 Okumura, A., Unoki-Kubota, H., Matsushita, Y., Shiga, T., Moriyoshi, Y., Yamagoe, S., et al. 529 2013. Increased serum leukocyte cell-derived chemotaxin 2 (LECT2) levels in obesity and fatty liver. Biosci. Trends 7(6): 276-283. doi:10.5582/bst.2013.v7.6.276.

531

532

533

534

535

536

537

538

539

540

541

542

543

544

545

546

547

548

549

550

Potthoff, M.J., Kliewer, S.A., and Mangelsdorf, D.J. 2012. Endocrine fibroblast growth factors 15 / 19 and 21: from feast to famine. Genes Dev. 26(4): 312-324. doi:10.1101/gad.184788.111.

Saito, Y., Watanabe, Y., Saito, E., Honjoh, T., and Takahashi, K. 2001. Production and application of monoclonal antibodies to human selenoprotein P. J. Health Sci. 47(4): 346-352. doi:10.1248/jhs.47.346.

Sherk, V.D., Chrisman, C., Smith, J., Young, K.C., Singh, H., Bemben, M.G., et al. 2013. Acute bone marker responses to whole-body vibration and resistance exercise in young women. J. Clin. Densitom. 16(1): 104-109. doi:10.1038/jid.2014.371.

Slusher, A.L., Whitehurst, M., Zoeller, R.F., Mock, J.T., Maharaj, M., and Huang, C.-J. 2015. Attenuated fibroblast growth factor 21 response to acute aerobic exercise in obese individuals. Nutr. Metab. Cardiovasc. Dis. 25(9): 839-845. doi:10.1016/j.numecd.2015.06.002.

Sylow, L., Kleinert, M., Richter, E.A., and Jensen, T.E. 2017. Exercise-stimulated glucose uptake - regulation and implications for glycaemic control. Nat. Rev. Endocrinol. 13(3): 133-148. doi:10.1038/nrendo.2016.162.

Tanaka, M., Saito, Y., Misu, H., Kato, S., Kita, Y., Takeshita, Y., et al. 2016. Development of a sol particle homogeneous immunoassay for measuring full-length selenoprotein $\mathrm{P}$ in human serum. J. Clin. Lab. Anal. 30(2): 114-122. doi:10.1002/jcla.21824.

Taniguchi, H., Tanisawa, K., Sun, X., Kubo, T., and Higuchi, M. 2016. Endurance exercise 
reduces hepatic fat content and serum fibroblast growth factor 21 levels in elderly men. J. Clin. Endocrinol. Metab. 101(1): 191-198. doi:10.1210/jc.2015-3308.

553 Trepanowski, J.F., Mey, J., and Varady, K.A. 2014. Fetuin-A: a novel link between obesity 554 and related complications. Int. J. Obes. (Lond). 39(5): 734-741. doi:10.1038/ijo.2014.203.

556 Ueland, T., Aukrust, P., Aakhus, S., Smith, C., Endresen, K., Birkeland, K.I., et al. 2012. Activin A and cardiovascular disease in type 2 diabetes mellitus. Diab. Vasc. Dis. Res. 9(3): 234-237. doi:10.1177/1479164111431171.

Wildman, R.P., Muntner, P., Reynolds, K., McGinn, A.P., Rajpathak, S., Wylie-Rosett, et al. 2008. The obese without cardiometabolic risk factor clustering and the normal weight with cardiometabolic risk factor clustering. Arch. Intern. Med. 168(15): 1617-1624.

562

Xu, Y., Xu, M., Bi, Y., Song, A., Huang, Y., Liu, Y., et al. 2011. Serum fetuin-A is correlated 563 with metabolic syndrome in middle-aged and elderly Chinese. Atherosclerosis, 216(1): 180-186. doi:10.1016/j.atherosclerosis.2011.01.020.

Yang, S.J., Hwang, S.Y., Choi, H.Y., Yoo, H.J., Seo, J.A., Kim, S.G., et al. 2011. Serum 566 selenoprotein $\mathrm{P}$ levels in patients with type 2 diabetes and prediabetes: Implications for insulin resistance, inflammation, and atherosclerosis. J. Clin. Endocrinol. Metab. 96(8): 1325-1329. doi:10.1210/jc.2011-0620.

Zhang, X., Yeung, D.C.Y., Karpisek, M., Stejskal, D., Zhou, Z., Liu, F., et al. 2008. Serum FGF21 levels are increased in obesity and are independently associated with the metabolic syndrome in humans. Diabetes, 57(5): 1246-1253. doi:10.2337/db07-1476. 


\section{Tables}

\section{Table 1 - Participant Characteristics}

\begin{tabular}{|c|c|c|c|c|c|c|}
\hline \multirow[b]{2}{*}{ Anthropometry } & \multicolumn{3}{|c|}{$\begin{array}{l}\text { Normal weight } \\
(\mathrm{n}=11)\end{array}$} & \multicolumn{3}{|c|}{$\begin{array}{c}\text { Overweight/obese } \\
(n=11) \\
\end{array}$} \\
\hline & & & & & & \\
\hline $\operatorname{BMI}\left(\mathrm{kg} \cdot \mathrm{m}^{-2}\right)^{a}$ & \multicolumn{3}{|c|}{$23.4(1.6)$} & \multicolumn{3}{|c|}{$29.2(4.5)^{\ddagger}$} \\
\hline Age (years) & 36 & \pm & 15 & 45 & \pm & 14 \\
\hline Body weight (kg) & 69.8 & \pm & 1.5 & 92.3 & \pm & $3.4^{\ddagger}$ \\
\hline Body fat (\%) & 16.9 & \pm & 3.6 & 26.4 & \pm & $4.0^{\ddagger}$ \\
\hline Waist circumference $(\mathrm{cm})$ & 81.6 & \pm & 5.3 & 96.0 & \pm & $7.8^{\ddagger}$ \\
\hline \multicolumn{7}{|l|}{ Cardiorespiratory Fitness } \\
\hline Absolute $\dot{V} \mathrm{O}_{2}$ peak $\left(\mathrm{L} \cdot \mathrm{min}^{-1}\right)$ & 3.46 & \pm & 0.74 & 3.21 & \pm & 1.21 \\
\hline Relative $\dot{V} \mathrm{O}_{2}$ peak $\left(\mathrm{mL} \cdot \mathrm{kg} \mathrm{BW}^{-1} \cdot \mathrm{min}^{-1}\right)$ & 50.1 & \pm & 11.9 & 38.5 & \pm & $9.7^{\dagger}$ \\
\hline \multicolumn{7}{|l|}{ Circulating Metabolic Risk Factors } \\
\hline Total cholesterol $\left(\mathrm{mmol} \cdot \mathrm{L}^{-1}\right)$ & 4.12 & \pm & 0.73 & 4.91 & \pm & $0.89 *$ \\
\hline $\mathrm{TAG}\left(\mathrm{mmol} \cdot \mathrm{L}^{-1}\right)$ & 1.04 & \pm & 0.15 & 1.82 & \pm & $0.24^{\dagger}$ \\
\hline $\operatorname{NEFA}\left(\mathrm{mmol} \cdot \mathrm{L}^{-1}\right)$ & 0.39 & \pm & 0.18 & 0.58 & \pm & $0.14^{\dagger}$ \\
\hline FPG $\left(\mathrm{mmol} \cdot \mathrm{L}^{-1}\right)$ & 4.9 & \pm & 0.2 & 5.0 & \pm & 0.3 \\
\hline FPI $\left(\mathrm{pmol} \cdot \mathrm{L}^{-1}\right)$ & 30.0 & \pm & 12.2 & 37.1 & \pm & 19.3 \\
\hline \multicolumn{7}{|l|}{ Insulin Sensitivity } \\
\hline HOMA-IR & 0.95 & \pm & 0.39 & 1.21 & \pm & 0.67 \\
\hline Adipo-IR & 12.57 & \pm & 8.99 & 21.98 & \pm & 13.01 \\
\hline \multicolumn{7}{|l|}{ Hepatokines } \\
\hline FGF21 $\left(\mathrm{pg} \cdot \mathrm{mL}^{-1}\right)$ & 83 & \pm & 55 & 190 & \pm & 74 \\
\hline Follistatin $\left(\mathrm{pg} \cdot \mathrm{mL}^{-1}\right)$ & 795 & \pm & 257 & 670 & \pm & 154 \\
\hline LECT2 $\left(\mathrm{ng} \cdot \mathrm{mL}^{-1}\right)$ & 31 & \pm & 10 & 48 & \pm & $17^{\dagger}$ \\
\hline Fetuin-A $\left(\mu \mathrm{g} \cdot \mathrm{mL}^{-1}\right)$ & 541 & \pm & 137 & 497 & \pm & 99 \\
\hline $\operatorname{SeP}\left(\mu \mathrm{g} \cdot \mathrm{mL}^{-1}\right)$ & 3.01 & \pm & 0.39 & 2.81 & \pm & 0.30 \\
\hline
\end{tabular}


575 BMI: Body mass index; $\dot{V} \mathrm{O}_{2}$ peak: Peak oxygen uptake; TAG: Fasted triacylglycerol; NEFA:

576 Fasted non-esterified fatty acids; FPG: Fasted plasma glucose; FPI: Fasted plasma insulin;

577 HOMA-IR: Homeostatic model assessment of insulin resistance; Adipo-IR: Adipose tissue

578 insulin resistance index; FGF21: Fibroblast growth factor 21; LECT2: Leukocyte cell-derived

579 chemotaxin 2; SeP: Selenoprotein P. ${ }^{a}$ Heterogeneous variance between groups, non-

580 parametric analyses performed and data presented as median (interquartile range). All other

581 data presented as mean \pm SD. Symbols indicate statistically significant differences between

$582 \operatorname{groups}\left({ }^{*}<0.05 ;^{\dagger} \leq 0.01 ;{ }^{\dagger}<0.001\right)$. 
Table 2 - Exercise Characteristics

\begin{tabular}{|c|c|c|c|c|}
\hline \multirow[b]{2}{*}{ Treadmill speed $\left(\mathrm{km} \bullet \mathrm{h}^{-1}\right)$} & $\begin{array}{c}\text { Normal weight } \\
(\mathrm{n}=11)\end{array}$ & \multicolumn{3}{|c|}{$\begin{array}{c}\text { Overweight/obese } \\
(n=11)\end{array}$} \\
\hline & $7.6 \pm 1.0$ & 6.8 & \pm & $1.0^{*}$ \\
\hline$\dot{V} \mathrm{O}_{2}$ elicited $\left(\% \dot{V} \mathrm{O}_{2}\right.$ peak $)$ & $59.3 \pm 2.8$ & 57.9 & \pm & 2.3 \\
\hline Net energy expenditure $(\mathrm{kJ})$ & $2211 \pm 507$ & 2217 & \pm & 509 \\
\hline Heart rate (beats $\bullet \mathrm{min}^{-1}$ ) & $141 \pm 29$ & 139 & \pm & 19 \\
\hline Rate of perceived exertion (6-20) & $13 \pm 1$ & 12 & \pm & 1 \\
\hline
\end{tabular}

$584 \quad \dot{V} \mathrm{O}_{2}$ : oxygen uptake; $\dot{V} \mathrm{O}_{2}$ peak: peak oxygen uptake. Data presented as mean \pm SD. "indicates

585 a statistically significant difference between groups $(P<0.05)$. 
587 Figure 1 - Hepatokine responses in normal weight and overweight/obese groups.

588 Circulating plasma concentrations FGF21 (a-b), follistatin (c-d), LECT2 (e-f), fetuin-A (g-h)

589 and SeP (i-j) during control and exercise trials in both normal weight and overweight/obese

590 groups. Meals were provided at 1.5 and $4 \mathrm{~h}$. Exercise was performed between 0 and $1 \mathrm{~h}$ in the

591 exercise trial only. Data presented as mean \pm SEM. AUC represents the total area under the

592 concentration-time curve for the given experimental day. $P$-values denote significant main

593 effect of group irrespective or time or trial.

594

595 Figure 2 - Hepatokine exercise responses with groups combined to a single population.

596 Circulating plasma concentrations FGF21 (a-b), follistatin (c-d), LECT2 (e-f), fetuin-A (g-h)

597 and SeP (i-j) during control and exercise trials in the whole study population combined.

598 Meals were provided at 1.5 and $4 \mathrm{~h}$. Exercise was performed between 0 and $1 \mathrm{~h}$ in the 599 exercise trial only. Data presented as mean \pm SEM. AUC represents the total area under the 600 concentration-time curve for the given experimental day. * indicates significant difference 601 from control trial at the same time point (all $P \leq 0.02$ ). $P$-values on AUC plots denote 602 significant difference between control and exercise trials.

603

604 Figure 3 - Responses in NEFA, glucagon and insulin with groups combined to a single 605 population. Circulating plasma concentrations (a) NEFA (b) glucagon (c) insulin and (d) the 606 glucagon to insulin ratio during control and exercise trials in the whole study population 607 combined. Meals were provided at 1.5 and $4 \mathrm{~h}$. Exercise was performed between 0 and $1 \mathrm{~h}$ in 608 the exercise trial only. Data presented as mean \pm SEM. * indicates significant difference from 609 control trial at the same time point (all $P \leq 0.04)$.

610 
a)

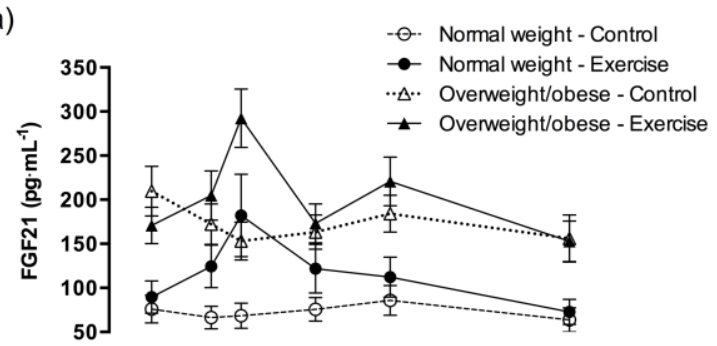

c)

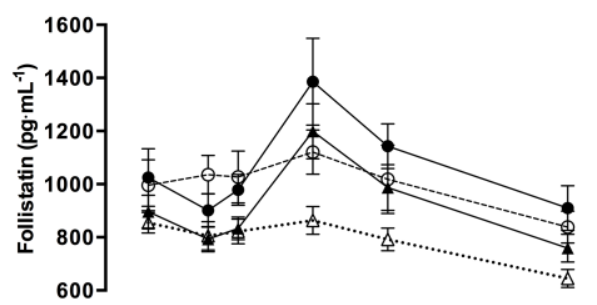

e)

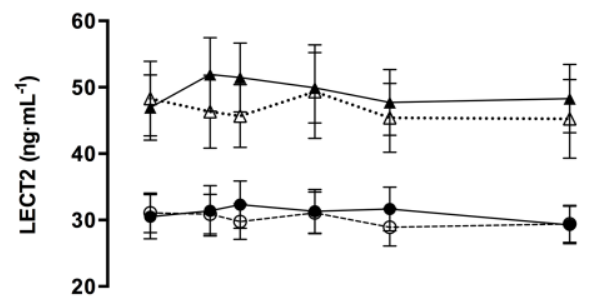

g)

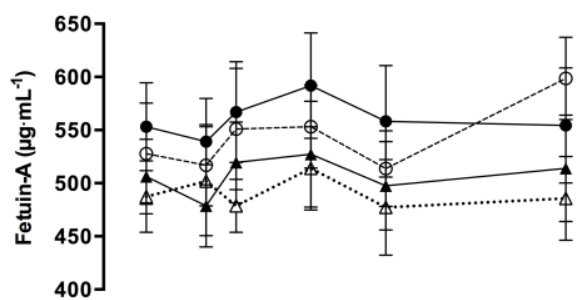

i)

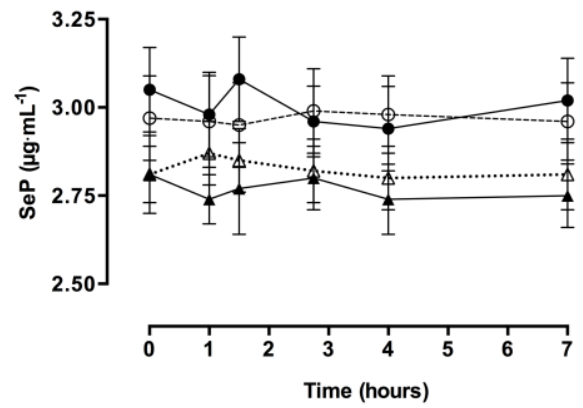

b)

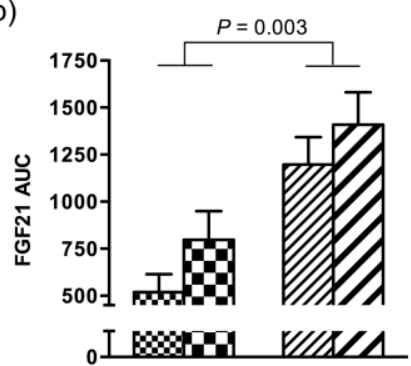

d)
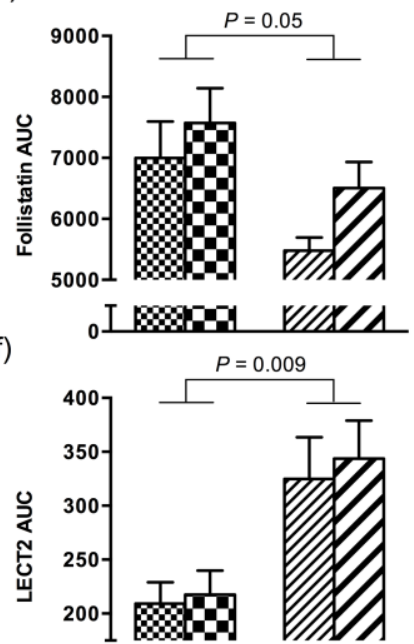

h)
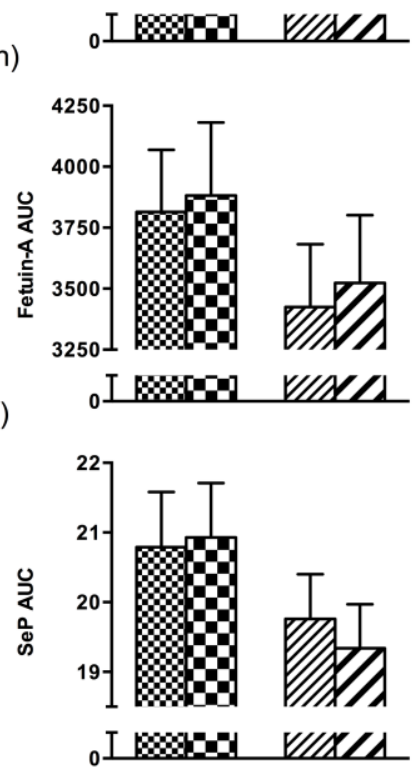
a)

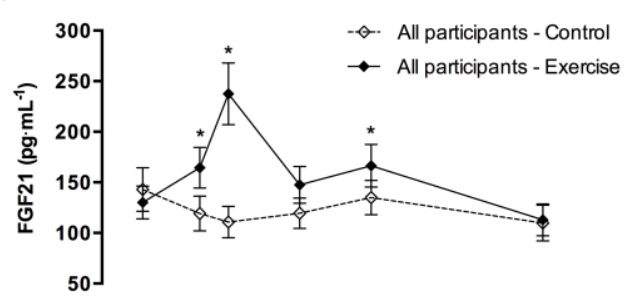

c)

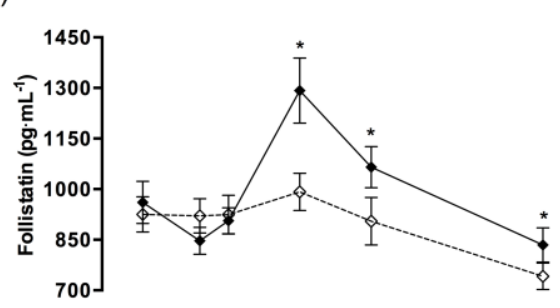

e)

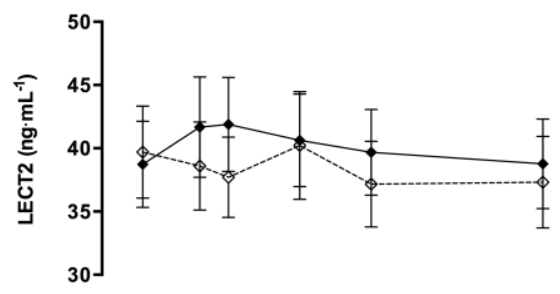

g)

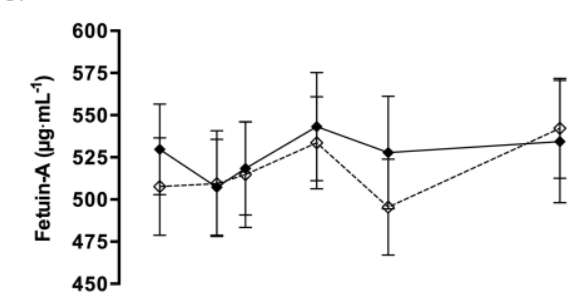

i)

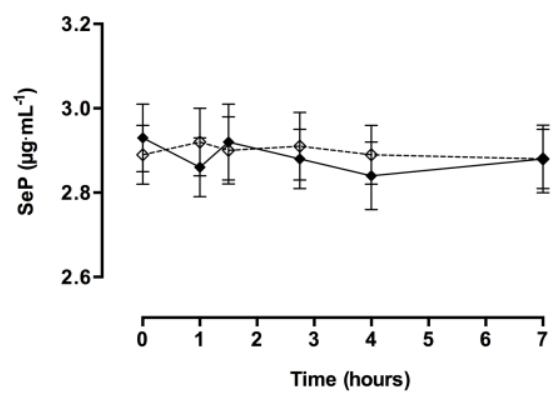

b)

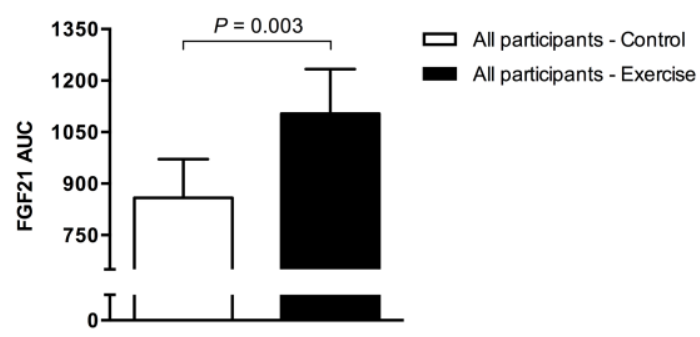

d)

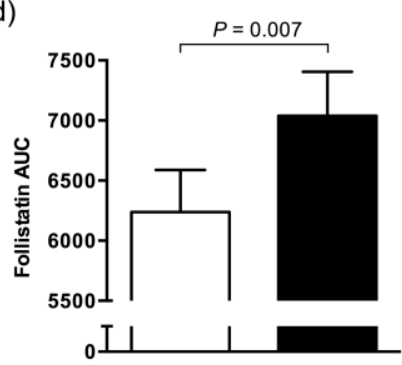

f)

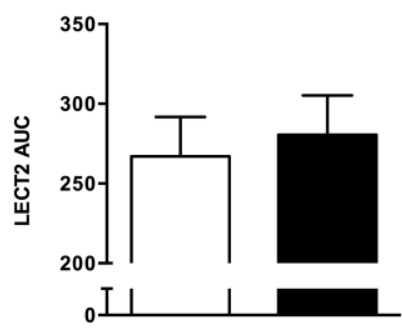

h)

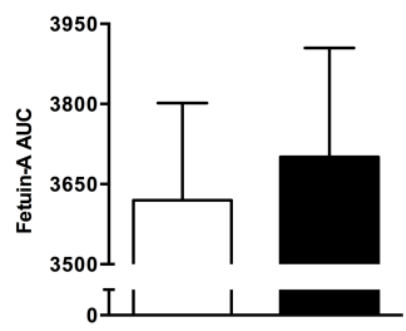

j)

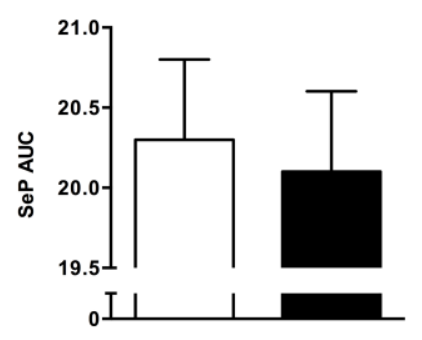


a)

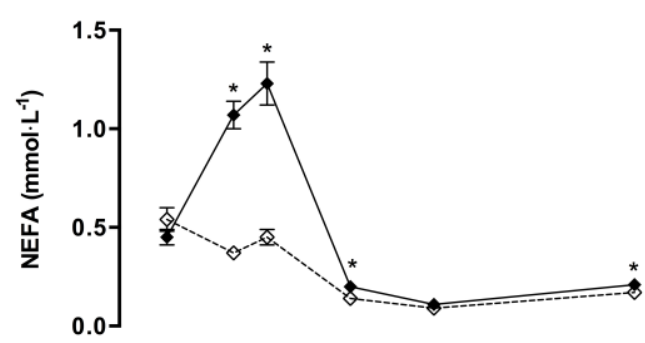

c)

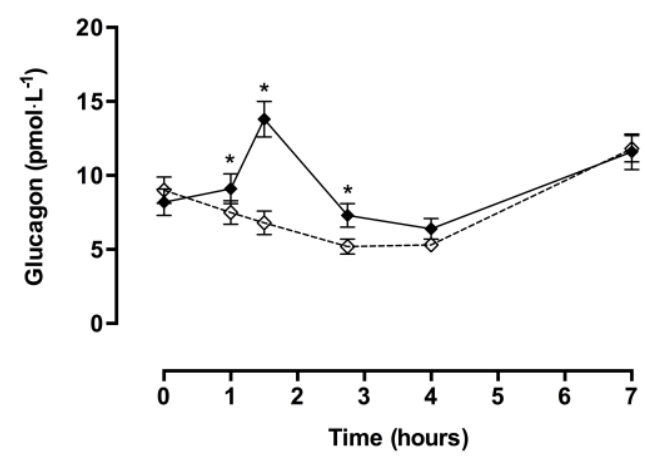

b)

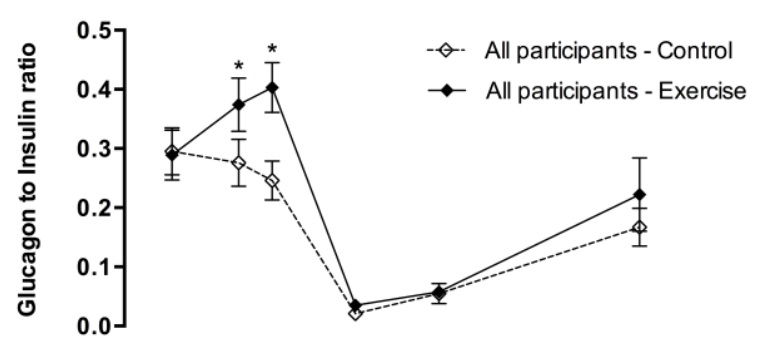

d)

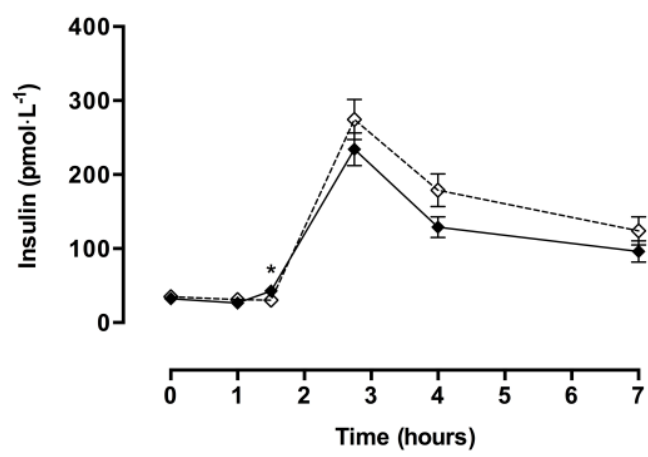

